DOI 10.4171/JEMS/253

F. Acquistapace · A. Díaz-Cano

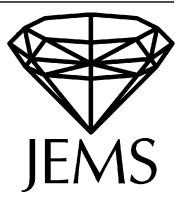

\title{
Divisors in global analytic sets
}

Received January 21, 2008 and in revised form January 8, 2009

\begin{abstract}
We prove that any divisor $Y$ of a global analytic set $X \subset \mathbb{R}^{n}$ has a generic equation, that is, there is an analytic function vanishing on $Y$ with multiplicity one along each irreducible component of $Y$. We also prove that there are functions with arbitrary multiplicities along $Y$. The main result states that if $X$ is pure dimensional, $Y$ is locally principal, $X \backslash Y$ is not connected and $Y$ represents the zero class in $\mathrm{H}_{q-1}^{\infty}\left(X, \mathbb{Z}_{2}\right)$ then the divisor $Y$ is globally principal.
\end{abstract}

Keywords. Real analytic sets, divisors

\section{Introduction}

In this paper we prove that any divisor $Y$ of a global analytic set $X \subset \mathbb{R}^{n}$ has a generic equation, that is, there is an analytic function vanishing on $Y$ with multiplicity one along each irreducible component of $Y$ (we refer to Section 2 below for the definition of divisor). Furthermore, it is proved that there are functions with arbitrary multiplicities along $Y$. Unfortunately we cannot infer, in general, that $Y$ is the zero set of this equation. Thus, one can ask under what conditions there is a global analytic function $g$ such that $Y=\operatorname{div}(g)$, in other words $g$ generates the ideal $\mathcal{J}_{Y}$. We find, at least when the space $X$ is of pure dimension, three conditions. The first one is an obvious local condition: the divisor must be locally principal. It is easy to find examples where it is not locally principal, even when the divisor has codimension 1 at every point. The second condition is a topological condition: a principal divisor $Y$ always has null fundamental class in the group $\mathrm{H}_{q-1}^{\infty}\left(X, \mathbb{Z}_{2}\right)$. Also a third topological condition is required, that $X \backslash Y$ is not connected. This is because a generator of $\mathcal{J}_{Y}$ cannot have constant sign, for instance at the points $y \in Y$ which are regular for both $X$ and $Y$.

We are able to prove that these conditions are sufficient for $Y$ to be principal when $X$ is pure dimensional, for instance when $X$ is a coherent analytic set.

We endow $X$ with its “best" coherent structure; by Cartan's Theorem B we have an isomorphism between the groups $H^{1}\left(X, \mathcal{O}^{*}\right)$ and $H^{1}(X, \mathbb{Z} / 2)$, so the conditions above

F. Acquistapace: Dipartimento di Matematica, Largo Pontecorvo 5, Università di Pisa, 56127 Pisa, Italy; e-mail: acquistf@dm.unipi.it

A. Díaz-Cano: Departamento de Álgebra, Facultad de Ciencias Matemáticas, Universidad Complutense de Madrid, 28040 Madrid, Spain; e-mail: adiaz@mat.ucm.es

Mathematics Subject Classification (2010): Primary 14P15; Secondary 32C05, 32C07 
imply that the line bundle defined by the local generators of $\mathcal{J}_{Y}$ is trivial in a neighbourhood of the set $X_{\max }=\left\{x \in X \mid \operatorname{dim} X_{x}=\operatorname{dim} X=q\right\}$, which, in turn, enables us to find a global generator on the same neighbourhood (see Theorem 3.4 below). As far as we know, this result was known only for analytic manifolds.

Using the principality conditions above and the notion of multiplicities along a divisor of analytic functions, which we will discuss in Section 1, for a coherent analytic set we prove an equivalent condition for an ideal of the type

$$
\prod_{i} \mathfrak{p}_{i}^{a_{i}}
$$

to be principal, where $\mathfrak{p}_{\mathfrak{i}}$ is a prime ideal in $\mathcal{O}(X)$, namely that the associated divisor $Y=\sum a_{i} Y_{i}$, where $Y_{i}$ is the zero set of $\mathfrak{p}_{\mathfrak{i}}$, has vanishing fundamental class $[Y]=0 \in$ $\mathrm{H}_{q-i}^{\infty}(X, \mathbb{Z} / 2)$, its support disconnects $X$ and for all $i$ the ideal sheaf $\mathfrak{p}_{\mathfrak{i}} \mathcal{O}_{X}$ is locally principal.

These results can be seen as an improvement of the solution of Cousin's Second Problem (see, for example, Chapter VIII of [GR65]) in the case of an analytic manifold $X$ such that $H^{2}(X, \mathbb{Z})=0$, since here we only assume that $X$ is pure dimensional.

The paper is organized as follows: in the first section we give some definitions and preliminary results on multiplicities. Section 2 is devoted to the problem of finding a generic equation of a divisor and a positive equation with arbitrary given multiplicities. Finally, in Section 3 we prove (for a pure dimensional space $X$ ) that the three conditions above imply $Y=\operatorname{div}(g)$.

\section{Multiplicities}

Let $X \subset \mathbb{R}^{n}$ be a global analytic set, i.e. the zero set of finitely many analytic functions in $\mathcal{O}_{\mathbb{R}^{n}}\left(\mathbb{R}^{n}\right)=\mathcal{O}_{n}\left(\mathbb{R}^{n}\right)$. Recall that a global analytic set admits coherent structures and admits complexifications, i.e. there exists a coherent ideal sheaf $\mathcal{F} \subset \mathcal{O}_{n}$ such that $X=\operatorname{Supp} \mathcal{O}_{n} / \mathcal{F}$ and there exists a complex analytic space $\widetilde{X}$ in a suitable complex Stein neighbourhood of $\mathbb{R}^{n}$ in $\mathbb{C}^{n}$ such that $\tilde{X} \cap \mathbb{R}^{n}=X$; moreover these three properties (to be global, to have a coherent structure and to be the real part of a complex analytic set) are equivalent (see Prop. 15 in [Car57] and [Tog67]).

One can prove that among the sheaves defining these coherent structures there is a largest one, which we will denote by $\mathcal{J}_{X}$; also among these complex analytic sets there is a smallest one, that we still denote $\widetilde{X}$. Moreover, for any real point $x, \mathcal{J}_{\widetilde{X}, x}=\mathcal{J}_{X, x} \otimes_{\mathbb{R}} \mathbb{C}$, i.e. they define on $X$ the same structure, the so called well-reduced structure (cf. [ABT75], [Gal76]). It can also be verified that $\mathcal{J}_{X}$ is precisely the sheaf generated by the ideal of analytic functions vanishing on $X$, namely $\mathcal{J}_{X, x}=I(X) \mathcal{O}_{n, x}$ where $I(X)=\{f \in$ $\mathcal{O}_{n}\left(\mathbb{R}^{n}\right) \mid f=0$ on $\left.X\right\}$ (cf. [BP04]).

We will call $\mathcal{O}_{X}=\mathcal{O}_{n} / \mathcal{J}_{X}$ the sheaf of analytic functions on $X$. The ring of global sections of this sheaf is $\mathcal{O}(X)=\mathcal{O}_{n}\left(\mathbb{R}^{n}\right) / I(X)$ and the ring $\mathcal{M}(X)$ of meromorphic functions on $X$ will be defined as the total ring of fractions of $\mathcal{O}(X)$. 
Note that the ideal $I(X)$ may be prime even if $X$ is not irreducible as analytic space. A classical example is

$$
X=\left\{x^{2}-\left(z^{2}-1\right) y^{2}=0\right\} \subset \mathbb{R}^{3} .
$$

The polynomial $p=x^{2}-\left(z^{2}-1\right) y^{2}$ is irreducible as analytic function and generates $I(X)$. Nevertheless, $X$ is the union of two analytic subspaces $X_{1}$ and $X_{2}$ that are not global, each one isomorphic to a Whitney umbrella.

So, from now on we shall call a global analytic set $X$ irreducible if it does not admit proper global analytic subsets of the same dimension, that is, if $I(X)$ is a prime ideal in $\mathcal{O}_{n}\left(\mathbb{R}^{n}\right)$.

Remark 1.1. The ideal sheaf $\mathcal{J}_{X}$ is not in general a sheaf of real ideals. More precisely $\sqrt[R]{\mathcal{J}_{X, x}} \neq \mathcal{J}_{X, x}$ if and only if there are some couples of complex conjugate components $Z_{x}, \overline{Z_{x}} \subset \widetilde{X}_{x}$ that intersect $X$ in the same real component $Z_{x} \cap \overline{Z_{x}}$ of dimension less than $\operatorname{dim} X$; for instance this is the case when $\operatorname{dim} X_{x}<\operatorname{dim} X=q$. The ideal sheaf $\sqrt[R]{\mathcal{J}_{X}}$, which is not coherent in general, is the ideal of all analytic germs vanishing at $X$. Take now $\bar{g} \in \mathcal{O}_{X, x}$ with $g \in \mathcal{O}_{n, x}$ and $g \notin \mathcal{J}_{X, x}$; then either $g \in \sqrt[R]{\mathcal{J}_{X, x}}$, or $g \notin \sqrt[R]{\mathcal{J}_{X, x}}$. In the first case $g$ vanishes on the set germ $X_{x}$; in the second one we may consider the sign of $\bar{g}$ on $X_{x}$ and the set germs $\{\bar{g} \geq 0\},\{\bar{g} \leq 0\}$ are defined as well as the set $\{\bar{g}=0\} \subset X_{x}$. This is because the sign of $g$ in a neighbourhood of $x$ in $X$ is the same as the sign of $g\left(\bmod \sqrt[R]{\mathcal{J}_{X, x}}\right)$ in the same neighbourhood, and the quotient map $\mathcal{O}_{n, x} \rightarrow \mathcal{O}_{n, x} / \sqrt[R]{\mathcal{J}_{X, x}}$ factorizes through $\mathcal{O}_{X, x}$. In particular $\mathcal{O}_{X, x}$ and $\mathcal{O}_{n, x} / \sqrt[R]{\mathcal{J}_{X, x}}$ have the same group of unities.

Now, let $Y \subset X$ be an irreducible global analytic subset of codimension 1 . We define the coherent sheaf of ideals $\mathcal{J}_{Y}=I(Y) \mathcal{O}_{X}$, where $I(Y)=\{f \in \mathcal{O}(X) \mid f=0$ on $Y\}$.

Suppose that at some point $x \in Y$ the ideal $\mathcal{J}_{Y, x}$ is principal, say $\mathcal{J}_{Y, x}=g \mathcal{O}_{X, x}$ for some $g \in \mathcal{O}_{X, x}$. Then the germ of any $f \in \mathcal{O}(X)$ at $x$ can be written as $f_{x}=g^{r} v$ for some nonnegative integer $r$ and some $v \notin \mathcal{J}_{Y, x}$.

Note that $r$ does not depend on the generator $g$. Indeed, suppose $f_{x}=h^{s} w$ for another generator $h$ and, say, $s \leq r$. Then there is a unit $u$ such that $h=u g$, hence $w u^{s} g^{s}=v g^{r}$, so $v g^{r-s}=w u^{s} \notin \mathcal{J}_{Y, x}$. This implies $s=r$.

Also, since $\mathcal{J}_{Y, x}$ is coherent the relation $f=u g^{r}$ holds in a neighbourhood of $x$, and $g$ generates $\mathcal{J}_{Y, y}$ for $y$ close to $x$ outside the zero set of $u$. In particular the integer $r$ is the same for $x$ and $y$.

The integer $r$ will be called the multiplicity of $f$ along $Y$ at the point $x$ and will be denoted as $m_{Y, x}(f)$. The multiplicity of a meromorphic function $f=f_{1} / f_{2} \in \mathcal{M}(X)$ where $f_{1}, f_{2} \in \mathcal{O}(X)$ (and $f_{2}$ is not a zero divisor of $\mathcal{O}(X)$ ) is defined as $m_{Y, x}(f)=$ $m_{Y, x}\left(f_{1}\right)-m_{Y, x}\left(f_{2}\right)$. It is straightforward to check that

$$
V_{Y, x}:=\left\{f \in \mathcal{M}(X) \mid m_{Y, x}(f) \geq 0\right\} \supset \mathcal{O}(X)
$$

is a discrete valuation ring.

Next, we want to prove that given $Y$ as above we can find a uniformizer $h \in \mathcal{O}(X)$ of $m_{Y}$ generating $\mathcal{J}_{Y, x}$ for almost all points $x \in Y$. We recall that a global analytic subset 
$W \subset X$ always admits a positive equation, that is, a nonnegative function $g \in \mathcal{O}(X)$ whose zero set is $\mathcal{Z}(g)=W$. One can take, for instance, $g=f_{1}^{2}+\cdots+f_{q}^{2}$, where $f_{1}, \ldots, f_{q} \in \mathcal{O}(X)$ are such that $W=\left\{f_{1}=0, \ldots, f_{q}=0\right\}$. Note that any such equation has multiplicity greater than 1 over $Y$. In particular $m_{Y, x}$, and consequently also $V_{Y, x}$, do not depend on the point $x \in Y$ provided $\mathcal{J}_{Y, x}$ is principal.

Lemma 1.2. Let $Y \subset X$ be an irreducible global analytic subset of codimension 1 such that $\mathcal{J}_{Y, p}$ is principal for some $p \in Y$. Then there is a uniformizer $h \in \mathcal{O}(X)$ of $m_{Y}$ such that $h_{x} \mathcal{O}_{X, x}=\mathcal{J}_{Y, x}$ for all $x \in Y$ off a real analytic set of codimension 1 in $Y$. Moreover, given any global analytic subset $Y^{\prime} \subset X$ such that $Y \not \subset Y^{\prime}$ the uniformizer $h$ can be chosen so that $\mathcal{Z}(h) \cap Y^{\prime}$ has no components of codimension 1 in $X$.

Proof. Assume $\mathcal{J}_{Y, p}=g \mathcal{O}_{X, x}$. By Cartan's Theorem A there are a finite number of global analytic functions on $X$ which generate the ideal $\mathcal{J}_{Y, p}$. At least one of these functions, call it $f$, has multiplicity 1 at $p$ along $Y$.

Let $\tilde{X}, \tilde{Y} \subset \Omega \subset \mathbb{C}^{n}$, where $\Omega$ is a Stein open neighbourhood of $\mathbb{R}^{n}$ in $\mathbb{C}^{n}$, be complexifications of $X$ and $Y$, respectively. Up to shrinking $\Omega$, the function $f \in \mathcal{O}(X)$ can be extended to a global analytic function on $\widetilde{X}$, which will still be called $f$. The ideal $\mathcal{J}_{\widetilde{Y}, p}=I(\widetilde{Y}) \mathcal{O}_{\widetilde{X}, p}$ is also principal generated by the same $g$, and $f_{p}=v_{p} g$, where $v_{p} \in \mathcal{O}_{\tilde{X}, p} \backslash \mathcal{J}_{\widetilde{Y}}, p$. Then, in a small complex neighbourhood $U$ of $p$ where $v$ is defined, $f_{x}$ generates $\mathcal{J}_{\tilde{Y}, x}$ for all $x \in \tilde{Y} \cap U \backslash\{v=0\}$. This last set is not empty, because $\tilde{Y}$ is pure dimensional; so, the set of points at which $f_{x}$ is a generator of $\mathcal{J}_{Y, x}$ is not empty.

Consider the coherent sheaf of ideals $\mathcal{J}$ defined by $\mathcal{J}_{x}=\left(f_{x} \mathcal{O}_{\widetilde{X}, x}: \mathcal{J}_{\widetilde{Y}_{, x}}\right)$, where $x \in \Omega$, that is, $h_{x} \in \mathcal{J}_{x}$ if and only if $h_{x} \mathcal{J}_{\widetilde{Y}, x} \subset f_{x} \mathcal{O}_{\widetilde{X}, x}$. Thus $\mathcal{J}_{x}=\mathcal{O}_{\tilde{X}, x}$ if and only if $f_{x}$ generates $\mathcal{J}_{\tilde{Y}, x}$. Therefore, the support

$$
\operatorname{supp}\left(\mathcal{O}_{\tilde{X}} / \mathcal{J}\right)=\left\{x \in \widetilde{X} \mid f_{x} \text { does not generate } \mathcal{J}_{\widetilde{Y}, x}\right\}
$$

is a closed analytic set $\widetilde{W}$ which does not contain $\tilde{Y}$. As $\widetilde{Y}$ is irreducible, $\widetilde{Y} \cap \widetilde{W}$ has codimension at least 1 in $\widetilde{Y}$. Hence, $f_{x}$ generates $\mathcal{J}_{\tilde{Y}, x}$ for all $x \in \widetilde{Y} \backslash \widetilde{W}$. Then also $f_{x}$ generates $\mathcal{J}_{Y, x}$ for all $x \in Y \backslash W$, where $W=\tilde{W} \cap \mathbb{R}^{n}$.

Note that $W \cap Y$ is a subset of codimension at least 1 in $Y$. For suppose that $W \gtrsim Y_{\max }$, where $Y_{\max }$ denotes the part of maximal dimension of $Y$. Then $W \supset Y$ and so $\widetilde{W} \supset Y$. But as $\widetilde{Y}$ is the complexification of $Y$, this in turn would imply $\widetilde{W} \supset \widetilde{Y}$, which is a contradiction.

Now, let $Y^{\prime}$ be any analytic set not containing $Y$. Take positive equations $f_{Y}, f_{Y^{\prime}} \in$ $\mathcal{O}(X)$ of $Y$ and $Y^{\prime}$, respectively. Then $\bar{f}=f_{Y^{\prime}} f+f_{Y}$ has the required properties.

Thus we can just write $m_{Y}$ and $V_{Y}$ for the multiplicity along $Y$ and its valuation ring. The next proposition gives another characterization of $V_{Y}$.

Proposition 1.3. Let $Y \subset X$ be an irreducible global analytic subset of codimension 1 such that $Y \cap \operatorname{Reg} X \neq \emptyset$. Then $V_{Y}=\mathcal{O}(X)_{I(Y)}$. In particular, $m_{Y}$ is a real valuation.

Proof. First of all, it is easy to check that $V_{Y} \supset \mathcal{O}(X)_{I(Y)}$. 
To prove the other inclusion, take some point $x \in \operatorname{Reg} Y \cap \operatorname{Reg} X$, which exists, since otherwise $\operatorname{Reg} Y \subset \operatorname{Sing} X$ and then $Y \subset \operatorname{Sing} X$. Let $\mathfrak{m}_{x} \subset \mathcal{O}(X)$ be the ideal of analytic functions on $X$ vanishing at $x$. As $\mathfrak{m}_{x} \supset I(Y)$, we have $\mathcal{O}(X)_{I(Y)}=\left(\mathcal{O}(X)_{\mathfrak{m}_{x}}\right)_{I(Y)}$. The ring $\mathcal{O}(X)_{\mathfrak{m}_{x}}$ is regular (cf. [ABR96, Proposition VIII.4.4]), so its localization at $I(Y) \mathcal{O}(X)_{\mathfrak{m}_{x}}$, which is a prime ideal of height one, is a discrete valuation ring. Hence, $\mathcal{O}(X)_{I(Y)} \supset V_{Y}$.

Finally, note that the residue field of $\mathcal{O}(X)_{I(Y)}$ is the field of meromorphic functions on $Y$, which is a real field.

\section{Divisors}

Let $X$ be a global analytic set in $\mathbb{R}^{n}$ as before. Set $q=\operatorname{dim} X$.

Definition 2.1. Let $\left\{Y_{i}\right\}_{i \in J}$ be a locally finite family of global irreducible analytic subsets of $X$, with for every $i, \operatorname{dim} Y_{i}=q-1$ and $Y_{i} \cap \operatorname{Reg} X \neq \emptyset$. A divisor in $X$ is the (formal) sum

$$
\sum_{i \in J} n_{i} Y_{i}
$$

where $n_{i} \in \mathbb{Z}$. The divisor is called reduced if $n_{i}=1$ for all $i$ and positive when $n_{i}>0$. The support of a divisor is the global analytic set $Y=\bigcup_{i} Y_{i}$. It is a global analytic subset of $X$, because the family $\left\{Y_{i}\right\}_{i \in J}$ is locally finite. The $Y_{i}$ in the family are called components of the divisor.

Finally, we say that two divisors $Y, Y^{\prime}$ are coprime if their supports do not share any irreducible component.

The set $\mathcal{D}$ of divisors has a natural structure of abelian group.

The multiplicities $m_{Y_{i}}$ along the components of a divisor are well defined. We shall say that $Y=\sum_{i \in J} n_{i} Y_{i}$ is the divisor of an analytic function $g$ and we shall write $Y=\operatorname{div}(g)$ if $m_{Y_{i}}(g)=n_{i}$ and the zero set of $g$ is the support of $Y$. In this case we shall call $Y$ principal.

Let $Y$ be (the support of) a divisor. Now by classical results on triangulations (cf. [Łoj64]), we may find a locally finite triangulation of the couple $(X, Y)$; this means that we have a simplicial complex $K$, together with a subcomplex $K_{Y}$, and a homeomorphism $f: K \rightarrow X$ such that $f\left(K_{Y}\right)=Y$ and for each simplex $\sigma$ of $K$, the restriction $\left.f\right|_{\sigma}$ is an analytic isomorphism. So, for any $j$, we have isomorphisms $f_{*}: \mathrm{H}_{j}^{\infty}\left(K, \mathbb{Z}_{2}\right) \rightarrow$ $\mathrm{H}_{j}^{\infty}\left(X, \mathbb{Z}_{2}\right)$.

Here $\mathrm{H}_{j}^{\infty}\left(X, \mathbb{Z}_{2}\right)$ is the homology group based on infinite chains; for the definition and generalities on the groups $\mathrm{H}_{j}^{\infty}\left(X, \mathbb{Z}_{2}\right)$ we refer to [Mas78].

Also, by the construction above, each component $Y_{i}$ of the divisor defines in a natural way an element $\left[Y_{i}\right]$ in the group $\mathrm{H}_{q-1}^{\infty}\left(X, \mathbb{Z}_{2}\right)$; note that a real analytic set carries a fundamental class (cf. [BH61]). Since any two such triangulations are PL-equivalent by Hauptvermutung (cf. [SY84]), this fact allows one to define a group homomorphism

$$
\mathcal{D} \rightarrow \mathrm{H}_{q-1}^{\infty}\left(X, \mathbb{Z}_{2}\right)
$$

sending the divisor $Y$ to the class $\sum_{i} n_{i}\left[Y_{i}\right]$. 
From now on we shall use the same symbol for both a divisor and its support when there is no risk of confusion.

We shall find for any reduced divisor $Y$ of $X$ what we will call a generic equation, that is, we shall find an analytic function $h$ vanishing on $Y$ with multiplicity 1 along each component $Y_{i}$ of $Y$.

Theorem 2.2 (Generic equation). Let $Y=\sum Y_{i}$ be a reduced divisor of $X$. Then there exists $h \in \mathcal{O}(X)$ such that $m_{Y_{i}}(h)=1$ for all $i \in I$. In particular, $h$ changes sign at every point of maximal dimension of $Y$ and it is a local generator of $\mathcal{J}_{Y, x}$ for all $x \in Y$ off an analytic set of codimension 1 in $Y$.

Moreover, given any global analytic subset $W \subset X$ not containing any component of $Y$ the function $h$ can be so chosen that $\mathcal{Z}(h) \cap W$ has codimension at least 2 in $X$.

Proof. For each $x \in X$ we write $J_{x}$ for the finite set of indices $i \in J$ such that $x \in Y_{i}$. Then we define the coherent sheaf $\mathcal{J}$ of ideals by

$$
\mathcal{J}_{x}=\left(\prod_{i \in J_{x}} h_{i, x}, \prod_{i \in J_{x}} g_{Y_{i}, x}\right),
$$

where each $h_{i}$ is a uniformizer of $m_{Y_{i}}$ not vanishing on $Y_{j}$, for $j \neq i$ (cf. Lemma 1.2) and $g_{Y_{i}}$ is a positive equation of $Y_{i}$. Since for every $x, \partial_{x}$ is generated by two functions, the sheaf $\mathcal{J}$ is globally generated by finitely many global sections $f_{1}, \ldots, f_{r}$ (cf. [Coe67]). Note that for each $Y_{i}$ at least one $f_{j}$ has multiplicity one along $Y_{i}$.

Set $I_{0}=\emptyset$ and define $I_{j}=\left\{i \in I \mid m_{Y_{i}}\left(f_{j}\right)=1\right\} \backslash \bigcup_{t=0}^{j-1} I_{t}$. We define the functions $f_{j}^{\prime}=f_{j}+e_{j}, j=1, \ldots, r$, where $e_{j}$ is a positive equation of $\bigcup_{i \in I_{j}} Y_{i}$.

Note that $\mathcal{Z}\left(f_{j}^{\prime}\right)=\bigcup_{i \in I_{j}} Y_{i}$ and $m_{Y_{i}}\left(f_{j}^{\prime}\right)=1$ for $i \in I_{j}$. Moreover for each $Y_{i}$ there is exactly one $f_{j}^{\prime}$ vanishing on $Y_{i}$. So $f=f_{1}^{\prime} \ldots f_{r}^{\prime}$ has multiplicity one along each $Y_{i}$.

Finally, as in the proof of Lemma 1.2, if $g_{Y}, g_{W} \in \mathcal{O}(X)$ are positive equations of $Y$ and $W$, respectively, then the zero set of $\bar{f}=g_{W} f+g_{Y}$ intersects $W$ along a set of codimension at least 2 .

This theorem says, in particular, that the zero set of $h$ can be written as $Y \cup Y^{\prime}$ for some analytic set $Y^{\prime}$. In general we can say little about the set $Y^{\prime}$ of "extra" zeroes of the function $h$, except that, if it is a divisor, it is coprime with $Y$ and can be chosen coprime with any divisor $W$ fixed in advance.

Thus, two questions arise. Is it possible to find a generic equation of $Y$ being a local generator of $\mathcal{J}_{Y, x}$ at every $x \in Y$ ? And, what can be said about $Y^{\prime}$ ? In the next section we will answer these questions under some additional hypotheses on the sheaf $\mathcal{J}_{Y}$ and on the space $X$.

If $Y$ is a divisor, then a positive equation of $Y$ has even multiplicity along each component $Y_{i}$ of $Y$. In the next theorem we show that given any sequence $\left\{m_{i}=2 n_{i}\right\}_{i \in I}$ of even positive integers we can find a positive equation of $Y$ with multiplicity precisely $m_{i}$ along each $Y_{i}$.

Theorem 2.3 (Positive equation). Let $Y=\sum 2 n_{i} Y_{i}$ be a positive even divisor. Then there is a positive analytic function $h$ such that $Y=\operatorname{div}(h)$. 
Proof. With the same notations of the previous theorem we define the coherent sheaf

$$
\mathcal{\partial}_{x}=\left(\prod_{i \in J_{x}} h_{i, x}^{n_{i}}, \prod_{i \in J_{x}} g_{Y_{i}, x}^{n_{i}}\right) .
$$

Again by [Coe67] this sheaf is generated by a finite number of global sections $f_{1}, \ldots, f_{r}$. Let $h=f_{1}^{2}+\cdots+f_{r}^{2}$. It is straightforward to see that $h$ is a positive equation of $Y=\bigcup Y_{i}$.

Now, for a given $Y_{i}$ take some point $x \in Y_{i} \backslash \bigcup_{j \neq i} Y_{j}$ such that $h_{i, x}$ generates $\mathcal{J}_{Y_{i}, x}$. Then $h_{i, x}^{n_{i}}$ generates $\partial_{x}$, so $\left(h_{i, x}^{n_{i}}\right)=\partial_{x}=\left(f_{1, x}, \ldots, f_{r, x}\right)$. Thus, $m_{Y_{i}}\left(f_{\ell}\right) \geq n_{i}$ for all $\ell=1, \ldots, r$ and $m_{Y_{i}}\left(f_{k}\right)=n_{i}$ for some $k$. As $m_{Y_{i}}$ is a real valuation, we have $m_{Y_{i}}(h)=2 \min _{\ell}\left\{m_{Y_{i}}\left(f_{\ell}\right)\right\}=2 n_{i}$.

As a corollary of the last two theorems, we prove that for any divisor $Y=\sum_{i} n_{i} Y_{i}$ there is a meromorphic function $f$ such that $m_{Y_{i}}(f)=n_{i}$ for each $i \in I$. But note again that, unless all multiplicities are even, the set of points where $f$ is zero or not analytic can be strictly larger than supp $Y$.

Corollary 2.4. Let $Y=\sum m_{i} Y_{i}$ be a divisor in $X$ where $\left\{m_{i}\right\}$ is any sequence of integers. Then there is $f \in \mathcal{M}(X)$ such that $m_{Y_{i}}(f)=m_{i}$ for all $i \in I$.

Proof. Write $m_{i}=2 n_{i}$ or $m_{i}=2 n_{i}+1$ according to the parity of $m_{i}$. By the theorem above, there is a sum of squares $h_{-} \in \mathcal{O}(X)$ such that $m_{Y_{i}}\left(h_{-}\right)=2\left|n_{i}\right|$ for all $i \in I$ such that $n_{i}<0$ with $\mathcal{Z}\left(h_{-}\right)=\bigcup_{n_{i}<0} Y_{i}$. Similarly there is a sum of squares $h_{+} \in \mathcal{O}(X)$ such that $m_{Y_{i}}\left(h_{+}\right)=2 n_{i}$ for all $i \in I$ such that $n_{i}>0$. Take $g \in \mathcal{O}(X)$ such that $m_{Y_{i}}(g)=1$ when $m_{i}$ is odd and not vanishing on any $Y_{i}$ such that $m_{i}$ is even.

Then $f=h_{+} g / h_{-}$has the required multiplicities. To check this just note that $m_{Y_{i}}(f)=m_{Y_{i}}\left(h_{+}\right)+m_{Y_{i}}(g)-m_{Y_{i}}\left(h_{-}\right)$.

A similar result has been proved in [ADR03] in the case of a real normal analytic surface $X$.

\section{Locally principal divisors}

Let $X$ be again a global analytic set in $\mathbb{R}^{n}$, of dimension $q$, well-reduced.

Let $Y \subset X$ be a reduced divisor; we are interested in the following question. Under what hypotheses is the ideal $I(Y)$ a principal ideal in $\mathcal{O}(X)$, that is, there is $g \in \mathcal{O}(X)$ such that $Y=\operatorname{div}(g)$ ? Note that if a global function $g$ generates $I(Y)$, then $X \backslash Y$ has at least two connected components and the set $\left\{y \in Y \mid \operatorname{dim}_{y} Y=\operatorname{dim}_{y} X-1\right\}$ bounds one of the regions where $g$ has a given sign. So, in order to have $Y=\operatorname{div}(g)$, the $\operatorname{divisor} Y$ must disconnect $X$ and the class $[Y]$ must vanish in the group $\mathrm{H}_{q-1}^{\infty}\left(X, \mathbb{Z}_{2}\right)$. However these conditions are not sufficient, as the following example shows.

Example 3.1. Consider the set

$$
X=\left\{(x, y, z) \in \mathbb{R}^{3} \mid z^{2}=x^{4}+y^{4}\right\}
$$


and put $Y=\{p \in X \mid x=0, z \geq 0\}$. Then $Y$ is a parabola and $[Y]=0$ in $\mathrm{H}_{q-1}^{\infty}\left(X, \mathbb{Z}^{2}\right)$. This is clear since $Y$ is the boundary of the open set $\{x>0, z>0\} \cap X$. However the ideal $I(Y) \mathcal{O}_{X, 0}$ is not principal; to see this one can apply [Mum88, Prop. 2, p. 384], or check it directly. If it were principal, $X \backslash Y$ would split into two principal open semianalytic sets, which is not the case, as proved in [Per01].

The previous example shows that another necessary condition for $Y$ to be principal is that the ideal sheaf $I(Y) \mathcal{O}_{X}$ is locally principal, that is, for any point $x \in X$ there is a function germ $f \in \mathcal{J}_{Y, x}$ that generates the stalk $\mathcal{J}_{Y, y}$ for any $y$ in a neighbourhood of $x$. So, from now on we will assume this condition. In case $X$ is not singular this condition follows from $[Y]=0$ as proved in [AB94]. In fact, it is shown there that in the nonsingular situation the condition $[Y]=0$ directly implies that $I(Y) \mathcal{O}_{X}$ is principal.

In order to generalise this result to the singular case we shall use some classical exact sequences of coherent sheaves and the vanishing of the cohomology with coefficients in a coherent sheaf.

As a first step we shall improve Theorem 2.2, in the sense that we will find a function $h$ vanishing with multiplicity one not only along $Y$ but along its whole zero set.

This result is proved in [BP04] for $X$ nonsingular.

Proposition 3.2. Let $X \subset \mathbb{R}^{n}$ be a global analytic set and let $Y \subset X$ be a reduced divisor such that $\mathcal{J}_{Y}$ is locally principal. Then there are an open neighbourhood $U$ of $X$ in $\mathbb{R}^{n}$ and global analytic hypersurfaces $W, W^{\prime}$ of $U$ such that $I\left(W \cup W^{\prime}\right)$ is generated by an analytic function $h \in \mathcal{O}_{n}(U), W \cap X=Y$ and $W^{\prime}$ is an analytic manifold transversal to $X$ and $Y$. Hence, if we set $Y^{\prime}=W^{\prime} \cap X$ then the ideal $I\left(Y \cup Y^{\prime}\right) \subset \mathcal{O}(X)$ is generated by $\left.h\right|_{X}$, and in particular $h$ generates the stalk $\mathcal{J}_{Y, x}$ at any point $x \in Y \backslash Y^{\prime}$.

Proof. Since $\mathcal{J}_{Y}$ is locally principal, for any $x$ there is a germ $f_{x} \in \mathcal{O}_{X, x}$ that generates $\mathcal{J}_{Y, y}$ for any $y$ in an open neighbourhood $U_{x}$ of $x$. So, refining the open covering $\left\{U_{x}\right\}_{x \in X}$, we can find a countable open covering $\left\{U_{i}\right\}$ of $X$ and analytic functions $f_{i}$ on $U_{i}$ such that $f_{i} \mathcal{O}_{X, x}=\mathcal{J}_{Y, x}$ for any $x \in U_{i}$; hence, $f_{i} / f_{j}$ is invertible on $U_{i} \cap U_{j}$. These functions define an analytic cocycle in $H^{1}\left(X, \mathcal{O}^{*}\right)$, that is, an analytic line bundle $\mathcal{F}$ on $X$. The collection $\left\{f_{i}\right\}$ defines a section of $\mathcal{F}$ vanishing exactly on $Y$.

We may find open sets $V_{i} \subset \mathbb{R}^{n}$ such that $V_{i} \cap X=U_{i}$. Since $\mathcal{O}_{X}$ is a coherent sheaf, each $f_{i}$ extends to an analytic function $F_{i}$ on the open set $V_{i}$ of $\mathbb{R}^{n}$.

Moreover, $f_{i} / f_{j}$ being invertible, after shrinking the $V_{i}$ 's we may assume that $F_{i} / F_{j}$ is invertible on $V_{i} \cap V_{j}$. So, $V=\bigcup_{i} V_{i}$ is an open neighbourhood of $X$ in $\mathbb{R}^{n}$ and the functions $F_{i} / F_{j}: V_{i} \cap V_{j} \rightarrow \mathcal{O}^{*}$ define an analytic line bundle $\mathcal{G}$ on $V$, extending $\mathcal{F}$. The collection $\left\{F_{i}\right\}$ defines an analytic section of $\mathcal{G}$ whose zero set $W$ cuts $X$ along $Y$.

We may find an analytic section $G=\left\{G_{i}\right\}$ of $\mathcal{G}$ transversal to the zero section (cf. [Tog80]), hence its zero set is an analytic manifold $W^{\prime}$ in $V$. We want to prove that $I\left(W \cup W^{\prime}\right)$ is principal in $\mathcal{O}_{n}(V)$. Arguing as in [BP04], consider the line bundle defined by $F_{i} G_{i}$ whose cocycle is $F_{i}^{2} F_{j}^{-2}$. We have to prove that this cocycle (and hence the line bundle) is trivial, i.e., $F_{i} G_{i} /\left(F_{j} G_{j}\right)=\lambda_{j}^{-1} \lambda_{i}$, where $\lambda_{i} \in \mathcal{O}_{n}^{*}\left(V_{i}\right)$; if so, $\left\{F_{i} G_{i} \lambda_{i}\right\}$ glue together and give a generator $h$ for $I\left(W \cup W^{\prime}\right)$. Define $Y^{\prime}=W^{\prime} \cap X$; then $\left.h\right|_{X}$ generates $I\left(Y \cup Y^{\prime}\right)$. 
Consider the exponential map and the associated usual exact sequence of coherent sheaves

$$
0 \rightarrow \mathcal{O}_{X} \rightarrow \mathcal{O}_{X}^{*} \rightarrow \mathcal{O}_{X}^{*} / \mathcal{O}_{X}^{+}=\mathbb{Z}_{2} \rightarrow 0 .
$$

Since $H^{i}\left(X, \mathcal{O}_{X}\right)=0$ for $i>0$, it induces an isomorphism between $H^{1}\left(X, \mathbb{Z}_{2}\right)$ and $H^{1}\left(X, \mathcal{O}_{X}^{*}\right)$. Under this isomorphism the image of a line bundle is the cocycle of the signs of its transition functions.

In our case $F_{i} G_{i} /\left(F_{j} G_{j}\right)=F_{i}^{2} / F_{j}^{2}$, so the line bundle is trivial and the proof is complete.

Remark 3.3. It is easy to check that if $\left\{Y_{i}\right\}_{i \in I}$ is a locally finite family of locally principal divisors then $\bigcup Y_{i}$ is also a locally principal divisor. On the other hand, we can have a locally principal divisor with some components which are not locally principal.

For example, take $X \subset \mathbb{R}^{3}$ to be the cone of equation $z^{2}=x^{2}+y^{2}$ and consider the divisor $Y=\{x=0\} \cap X$ which is locally principal with generator $g=x$. The divisor $Y$ splits into two straight lines $Y_{1}$ and $Y_{2}$ neither of which is locally principal.

We are ready to prove our main result.

Theorem 3.4. Let $X$ be a global analytic set in $\mathbb{R}^{n}$ and $q=\operatorname{dim} X$. Assume that the ideal $I(X) \subset \mathcal{O}\left(\mathbb{R}^{n}\right)$ is prime. Let $Y \subset X$ be a reduced divisor such that its ideal sheaf $\mathcal{J}_{Y}=I(Y) \mathcal{O}_{X}$ is locally principal; assume that $[Y]=0$ in $\mathrm{H}_{q-1}^{\infty}\left(X, \mathbb{Z}_{2}\right)$ and that $X \backslash Y$ is not connected. Then, there is an open neighbourhood $U$ of the set $X_{\max }=\{x \in X \mid$ $\left.\operatorname{dim}_{x} X=q\right\}$ and $g \in \mathcal{O}_{X}(U)$ such that $\mathcal{J}_{Y, x}=g \mathcal{O}_{X, x}$ for any $x \in U$; in particular, if $X$ has pure dimension $q$, then $Y=\operatorname{div}(g)$.

Proof. Arguing as in 3.2 we have to prove that there exists $U$ as stated such that the bundle $\mathcal{F}$ defined by $Y$ is trivial when restricted to $U$; to do this it is enough to find local generators $\left\{f_{i}\right\}$ of $\mathcal{J}_{Y}$ on a countable open covering $\left\{U_{i}\right\}$ of a neighbourhood of $X_{\max }$ such that $\left.f_{i}\right|_{U_{i} \cap U_{j}}$ and $\left.f_{j}\right|_{U_{i} \cap U_{j}}$ have the same sign.

Take a locally finite triangulation $f: K \rightarrow X$ of the couple $(X, Y)$; here $K$ is a simplicial complex, and there is a subcomplex $K_{Y}$ such that $f\left(K_{Y}\right)=Y$. In particular, for any $j$, we have isomorphisms $f_{*}: \mathrm{H}_{j}^{\infty}\left(K, \mathbb{Z}_{2}\right) \rightarrow \mathrm{H}_{j}^{\infty}\left(X, \mathbb{Z}_{2}\right)$.

The fact that $[Y]=0$ means that the union of all $q-1$ simplexes in $K_{Y}$ bounds some subcomplex $H$ of $K$. The boundary of the region $f(H) \subset X$ is the set $Y_{\max }$ of points in $Y$ where $Y$ has dimension $q-1$.

So, for each $j$ such that $U_{j} \cap X_{\max } \neq \emptyset$, we may choose local generators $g_{j} \in \mathcal{O}_{X}\left(U_{j}\right)$ in such a way that $g_{j}$ generates $\mathcal{J}_{Y}$ on $U_{j}$ and it is positive on $f(H) \cap U_{j} \backslash Y$, while if $f(H) \cap U_{j}=\emptyset$, we choose $g_{j}$ such that $g_{j} \geq 0$ when $U_{j}$ lies in the same connected component of $X \backslash Y$ as some component of $f(H)$ and $g_{j} \leq 0$ otherwise.

Hence $g_{i} / g_{j}>0$ on $U_{i} \cap U_{j}$, and $\mathcal{F}$ is trivial when restricted to $U$. This means that we can find analytic functions $\left\{\lambda_{i}\right\} \in \mathcal{O}^{*}\left(U_{i}\right)$ such that $g_{i} / g_{j}=\lambda_{j} / \lambda_{i}$. So, the sections $g_{i} \lambda_{i}: U_{i} \rightarrow \mathcal{O}_{X}$ satisfy $\left.g_{i} \lambda_{i}\right|_{U_{i} \cap U_{j}}=\left.g_{j} \lambda_{j}\right|_{U_{i} \cap U_{j}}$, that is, they define an analytic function $g$ on $U=\bigcup U_{i}$; by construction, $g_{x}$ generates $\mathcal{J}_{Y, x}$ for any $x \in U$.

Corollary 3.5. Under the hypothesis of Theorem 3.4 if $X$ is of pure dimension then $Y$ is principal. In particular, when $X$ is coherent, we have $Y=\operatorname{div}(g)$ for some $g \in \mathcal{O}(X)$. 
Remark 3.6. Note that when $X$ is a manifold, the condition $[Y]=0$ implies that $Y$ divides $X$ into two or more connected components and it is the boundary of some of them. Nevertheless this is not true in general, not even in the case of a coherent singular space $X$ : as an example one can consider $X$ to be a real 2-dimensional torus with one meridian collapsed to a point. One can easily write an analytic function on $\mathbb{R}^{3}$ with such a zero set. Take $Y$ to be any other meridian. Then $[Y]=0$, since it is homotopic to one point, and of course $Y$ is locally principal, but it cannot be principal because its complement is connected.

As a consequence of Theorem 3.4 we have the following analogue of a result by Shiota ([Shi81] ) that may be found in [BCR87, 12.4.1].

Corollary 3.7. Let $X$ be a global coherent analytic set in $\mathbb{R}^{n}$ and assume that the ideal $I(X) \subset \mathcal{O}\left(\mathbb{R}^{n}\right)$ is prime. Let $\mathfrak{p}_{i}, i \in \mathbb{N}$, be prime real ideals in $\mathcal{O}(X)$ of height 1 . Denote by $Y_{i}$ the associated divisor, i.e. the zero set of $\mathfrak{p}_{i}$, and assume that the family $\left\{Y_{i}\right\}_{i}$ is locally finite and that for any $i$ the ideal sheaf $\mathfrak{p}_{i} \mathcal{O}_{X}$ is locally principal. Then the ideal

$$
\prod_{i} \mathfrak{p}_{i}^{a_{i}}
$$

is principal if and only if the cycle $\sum_{i} a_{i}\left[Y_{i}\right]$ is zero in $\mathrm{H}_{q-1}^{\infty}\left(X, \mathbb{Z}_{2}\right)$ and $X \backslash \bigcup_{a_{i} \text { odd }} Y_{i}$ is not connected.

Proof. Since the family $\left\{Y_{i}\right\}_{i}$ of irreducible divisors is locally finite, the ideal sheaf $\prod_{i} \mathfrak{p}_{i}^{a_{i}} \mathcal{O}_{X}$ is also locally principal. Put $a_{i}=2 k_{i}$ or $a_{i}=2 k_{i}+1$ according to the parity of $a_{i}$. Split the class $\sum_{i} a_{i}\left[Y_{i}\right]$ as

$$
\sum_{i} 2 k_{i}\left[Y_{i}\right]+\sum_{a_{i}=2 k_{i}+1}\left[Y_{i}\right] .
$$

Note that the ideal sheaf $\mathcal{J}=\prod_{i} \mathfrak{p}_{i}^{2 k_{i}} \mathcal{O}_{X}$ is principal. In fact it is locally generated by a square, hence, arguing as in the proof of Theorem 3.4 its associated line bundle is trivial, which in turn implies that we can find a global section $g$ of $\mathcal{J}$ such that $g_{x}$ generates its stalk at any point $x \in X$. Also, $Y=\sum_{a_{i}=2 k_{i}+1} Y_{i}$ satisfies the hypothesis of Theorem 3.4 . so its ideal is principal, say generated by $f$. So, $f g$ generates $\prod_{i} \mathfrak{p}_{i}^{a_{i}}$ as desired.

The converse is clear.

Acknowledgments. The first author was partially supported by MURST, and the second author by Spanish GEOR MTM2008-00272 and Grupo UCM 910444. Both authors were partially supported by EC contract HPRN-CT-2001-00271 and HI2000-0127.

\section{References}

[AB94] Acquistapace, F., Broglia, F.: More about signatures and approximation. Geom. Dedicata 50, 107-116 (1994) Zbl 0822.14025 MR 1279880

[ABT75] Acquistapace, F., Broglia, F., Tognoli, A.: Sulla normalizzazione degli spazi analitici reali. Boll. Un. Mat. Ital. (4) 12, 26-36 (1975) Zbl 0318.32001 MR 0396993 
[ABR96] Andradas, C., Bröcker, L., Ruiz, J. M.: Constructible Sets in Real Geometry. Springer, Berlin (1996) Zbl 0873.140441037.32010

[ADR03] Andradas, C., Díaz-Cano, A., Ruiz, J. M.: The Artin-Lang property for normal real analytic surfaces. J. Reine Angew. Math. 556, 99-111 (2003) Zbl 1037.32010 MR 1971140

[BCR87] Bochnak, J., Coste, M., Roy, M.-F.: Géométrie algébrique réelle. Springer, Berlin (1987) Zbl 0633.14016 MR 0949442

[BH61] Borel, A., Haefliger, A.: La classe d'homologie fondamental d'un espace analytique. Bull. Soc. Math. France 89, 461-513 (1961) Zbl $0102.38502 \mid$ MR 0149503

[BP04] Broglia, F., Pieroni, F.: Separation of global semianalytic subsets of 2-dimensional analytic manifolds. Pacific J. Math. 214, 1-16 (2004) Zbl 1083.32006 MR 2039122

[Car57] Cartan, H.: Variétés analytiques réelles et variétés analytiques complexes. Bull. Soc. Math. France 85, 77-99 (1957) Zbl 0083.30502 MR 0094830

[Coe67] Coen, S.: Sul rango dei fasci coerenti. Boll. Un. Mat. Ital. 22, 373-383 (1967) Zbl 0164.38202 MR 0227467

[Fri67] Frisch, J.: Points de platitude d'un morphisme d'espaces analytiques complexes. Invent. Math. 4, 118-138 (1967) Zbl 0167.06803 MR 0222336

[Gal76] Galbiati, M.: Stratifications et ensemble de non-cohérence d'un espace analytique réel. Invent. Math. 34, 113-128 (1976) Zbl 0314.32006 MR 0417443

[Gua01] Guaraldo, F.: On the classification of real analytic fibre bundles. Math. Z. 237, 621-637 (2001) Zbl 0980.32003 MR 1845342

[GR65] Gunning, R., Rossi, H.: Analytic Functions of Several Complex Variables. Prentice-Hall, Englewood Cliffs, NJ (1965) Zbl 0141.08601 MR 0180696

[Łoj64] Łojasiewicz, S.: Triangulations of semi-analytic sets. Ann. Scuola Norm. Sup. Pisa 18, 449-474 (1964) Zbl 0128.17101 MR 0173265

[Mas78] Massey, W. S.: Homology and Cohomology Theory. Dekker, New York (1978) Zbl 0377.55004 MR 0488016

[Mum88] Mumford, D.: The Red Book of Varieties and Schemes. Lecture Notes in Math. 1358, Springer, Berlin (1988) Zbl 0658.14001 MR 0971985

[Nar66] Narasimhan, R.: Introduction to the Theory of Analytic Spaces. Lecture Notes in Math. 25, Springer, Berlin (1966) Zbl 0168.06003 MR 0217337

[Per01] Pernazza, L.: Basicness and separability with analytic and $C^{\infty}$ function germs. Tesi di Perfezionamento, Scuola Normale Superiore (2001)

[Shi81] Shiota, M.: Sur la factorialité de l'anneau des fonctions lisses rationnelles. C. R. Acad. Sci. Paris Sér. I 292, 67-70 (1981) Zbl 0489.14013 MR 0610150

[SY84] Shiota, M., Yokoi, M.: Triangulations of subanalytic sets and locally subanalytic manifolds. Trans. Amer. Math. Soc. 286, 727-750 (1984) Zbl 0527.57014 MR 0760983

[Ste74] Steenrod, N.: The Topology of Fibre Bundles. Princeton Univ. Press, Princeton NJ (1974) Zbl 0054.07103 MR 0039258

[Tog67] Tognoli, A.: Proprietà globali degli spazi analitici reali. Ann. Mat. Pura Appl. (4) 75, 143-218 (1967) Zbl 0158.33001 MR 0236418

[Tog80] Tognoli, A.: Une remarque sur les fibrés vectoriels analytiques et de Nash. C. R. Acad. Sci. Paris Sér. A-B 290, A321-A323 (1980) Zbl 0465.32006 MR 0567758 